\title{
Anterograde Transneuronal Viral Tracing of Central Viscerosensory Pathways in Rats
}

\author{
Linda Rinaman ${ }^{1}$ and Gary Schwartz ${ }^{2}$ \\ ${ }^{1}$ Department of Neuroscience, University of Pittsburgh, Pittsburgh, Pennsylvania 15260, and ²Bourne Behavioral Research Laboratory, Department of \\ Psychiatry, Weill Medical College Cornell University, White Plains, New York 10605
}

Previous studies demonstrated that strain H129 of herpes simplex virus-1 undergoes anterograde transneuronal transport in mice and primates after peripheral or central injection. In this study, H129 was used in rats to identify CNS regions that receive relayed viscerosensory inputs from the stomach wall. We also examined whether transneuronal viral transport in this model is exclusively anterograde. H129 or an established retrograde transneuronal viral tracer, pseudorabies virus (PRV), was injected into the ventral stomach wall in intact rats or in rats with previous subdiaphragmatic vagal sensory deafferentation. Rats were perfused with fixative 3-5 $\mathrm{d}$ later, and tissues were processed for immunocytochemical detection of transported virus. In intact rats, H129 was transported transneuronally via vagal and spinal viscerosensory neurons to postsynaptic target cells in the medullary dorsal vagal complex and thoracic dorsal horn, respectively, with subsequent transport to discrete regions of the medullary and pontine reticular formation, cerebellum, parabrachial nucleus, periaqueductal gray, thalamus, hypothalamus, amygdala, bed nucleus of the stria terminalis, and other central sites. Comparison of labeling patterns in intact and vagal deafferented rats indicated that $\mathrm{H} 129$ also produced first-order retrograde infection of autonomic neurons that project directly to virus injection sites, similar to PRV. Unlike PRV, however, H129 was not transported transneuronally in the retrograde direction from infected neurons to central sources of presynaptic input. We conclude that transneuronal transport of $\mathrm{H} 129$ occurs exclusively in the anterograde direction to reveal CNS regions that receive direct and relayed viscerosensory signals.

Key words: anterograde; dorsal horn; gastric; gastrointestinal; herpes; spinal; vagus; virus; viscerosensory; nucleus of the solitary tract

\section{Introduction}

Our current understanding of how central autonomic control circuits are organized owes much to the results of studies using neurotropic $\alpha$-herpes viruses for retrograde transneuronal tracing of multisynaptic pathways in rats (Strack et al., 1989; Loewy, 1995; Card, 1998). Such studies generally have used attenuated strains of pseudorabies virus (PRV) that move selectively across synapses in the retrograde direction. For example, the Bartha strain of PRV (Bartha, 1961) injected into the wall of the stomach (Card et al., 1993; Yang et al., 1999; Rinaman et al., 2000) or other visceral organs initially infects sympathetic and parasympathetic motor neurons and subsequently is transported retrogradely and transneuronally to infect pre-autonomic neurons within specific, circumscribed brainstem and forebrain regions.

A viral strain that is selectively transported across synapses in the anterograde direction would be equally useful for identifying CNS regions that receive direct and relayed sensory input from specific visceral organs. The H129 strain of herpes simplex virus-1 (Dix et al., 1983) undergoes anterograde transneuronal

Received Dec. 3, 2003; revised Jan. 30, 2004; accepted Feb. 6, 2004.

This work was supported by National Institutes of Health Grants MH59911 and DK47208. Victoria Maldovan and Judith Balcita-Pedicino provided expert technical assistance.

Correspondence should be addressed to Dr. Linda Rinaman, University of Pittsburgh, Department of Neuroscience, 446 Crawford Hall, Pittsburgh, PA 15260. E-mail: rinaman@pitt.edu.

D01:10.1523/JNEUROSCI.5329-03.2004

Copyright $\odot 2004$ Society for Neuroscience $\quad 0270-6474 / 04 / 242782-05 \$ 15.00 / 0$ transport in cebus monkeys after inoculation of primary motor cortex (Zemanick et al., 1991; Kelly and Strick, 2003) and in mice after inoculation of tooth pulp (Barnett et al., 1995) or the vitreous body of the eye (Sun et al., 1996). The present report documents our new finding that strain H129 also has utility as an anterograde transneuronal viral tracer in rats, effectively revealing CNS neurons that receive direct and relayed viscerosensory input from the stomach wall.

\section{Materials and Methods}

Experimental protocols conformed to current National Institutes of Health regulations and were approved by the University of Pittsburgh Institutional Animal Care and Use Committee.

Virus. Viral strain H129 was provided by Dr. Richard D. Dix (University of Arkansas for Medical Sciences, Little Rock, AR). PRV (Bartha strain) was provided by Dr. Lynn Enquist (Princeton University, Princeton, $\mathrm{NJ})$. Virus was maintained frozen at $-80^{\circ} \mathrm{C}$ in small aliquots until injection time.

Animals. Adult male Sprague Dawley rats (Harlan, Indianapolis, IN) were housed in a standard environment $\left(20-22^{\circ} \mathrm{C} ; 12 \mathrm{hr}\right.$ light beginning at 7:00 A.M.) with ad libitum access to water and pelleted Purina rat chow. Data from 34 rats are included in this report.

Subdiaphragmatic vagal deafferentation procedure. A subset of rats was subjected to subdiaphragmatic vagal sensory deafferentation before viral inoculation to determine the necessity of vagal sensory input to the nucleus of the solitary tract (NST) for central transneuronal viral transport from the stomach wall. Rats (200-225 gm) were deprived overnight of food but not water before vagal deafferentation surgery. Rats were anes- 
thetized by intramuscular injection of ketamine $(63 \mathrm{mg} / \mathrm{kg})$ and xylazine $(9.4 \mathrm{mg} / \mathrm{kg})$. The vagal deafferentation procedure has been described (Norgren and Smith, 1994; Schwartz et al., 1999) and was used here to eliminate vagal sensory innervation of the dorsal and ventral stomach wall while sparing unilateral vagal motor input to the ventral stomach wall. Deafferented rats were returned to their home cages after recovery from anesthesia. Because vagal deafferentation disrupts digestive functions, rats were given daily access to $25 \mathrm{ml}$ of a palatable liquid diet (Vanilla Ensure; Abbot Labs, Abbot Park, IL) in addition to chow to encourage voluntary food intake. Virus injections were performed $~ 3-6$ weeks later, after rats had resumed a normal growth curve.

Virus inoculation. Experimental manipulations involving virus were conducted in a Biosafety Level 2 laboratory. Non-food-deprived intact and vagal deafferented rats (260-325 gm) were anesthetized with halothane ( $2 \%$ in oxygen). The top left quadrant of the ventral abdomen was shaved and disinfected, and a $2.0-3.0 \mathrm{~cm}$ scalpel incision was made through the skin overlying the stomach. Surgical scissors were used to cut through the abdominal muscle layers, creating an opening just large enough to allow the stomach to be fully exteriorized. Using a $5.0 \mu \mathrm{l}$ Hamilton syringe with a sharpened beveled tip, a total volume of $8-10 \mu \mathrm{l}$ of either H129 $\left(1 \times 10^{10}\right.$ plaque forming units per milliliter $)$ or PRV $\left(2.0 \times 10^{8}\right.$ plaque forming units per milliliter) was injected tangentially between the muscle layers of the ventral stomach wall at multiple sites ( 1-2 $\mu$ l per site). After the final injection, the stomach surface was rinsed twice with sterile saline, blotted dry, and then returned to the abdominal cavity. The abdominal muscle incision was closed with silk sutures (Ethilon 6-0), and the skin incision was closed with stainless steel wound clips. Rats were returned to their home cages in the Biosafety facility after recovery from anesthesia. Rats infected with H129 or PRV remained asymptomatic until 4-5 d after virus injection, when many began to display adverse symptoms of infection (i.e., loss of body weight, hunched posture, ungroomed coat, and nasal and lacrimal excretions). Rats were killed either before or when such symptoms became apparent (i.e., $72-120 \mathrm{hr}$ after inoculation).

Perfusion fixation and tissue preparation. Rats were anesthetized with an overdose of sodium pentobarbital (Nembutal; $50 \mathrm{mg}$, i.p.) and killed by transcardiac perfusion with $50 \mathrm{ml}$ of $0.15 \mathrm{M} \mathrm{NaCl}$ followed by $500 \mathrm{ml}$ of $4 \%$ paraformaldehyde containing $1.4 \%$ L-lysine and $0.2 \%$ sodium meta-periodate (McLean and Nakane, 1974). Spinal cords, brains, celiac-superior mesenteric, and nodose ganglia were removed and postfixed at $4^{\circ} \mathrm{C}$ for $12-24 \mathrm{hr}$ and then cryoprotected at $4^{\circ} \mathrm{C}$ for $24 \mathrm{hr}$ in aqueous $20 \%$ sucrose. Coronal $35 \mu \mathrm{m}$ CNS sections were cut from the olfactory tubercle to the $\mathrm{L} 4$ level of the spinal cord using a freezing microtome. Sections were collected serially and stored at $-20^{\circ} \mathrm{C}$ in cryopreservant (Watson et al., 1986). Before immunoprocessing, sections were removed from cryopreservant and rinsed in several changes of buffer $(0.1 \mathrm{M}$ sodium phosphate, $\mathrm{pH}$ 7.2). Celiac-superior mesenteric and nodose ganglia were sectioned at $10 \mu \mathrm{m}$ intervals using a cryostat. Serial sections were collected onto SuperFrost Plus glass slides (Fisher Scientific, Pittsburgh, PA).

Antisera and immunocytochemical methods. All antisera were diluted in buffer containing 1\% donkey serum (Jackson ImmunoResearch, West Grove, PA) and 0.3\% Triton X-100 (Sigma, St. Louis, MO). For immunoperoxidase localization of H129 or PRV in brain and spinal cord, sections were incubated at $4^{\circ} \mathrm{C}$ for $48-72 \mathrm{hr}$ in rabbit anti-herpes simplex virus-1 antiserum (B-0114; 1:20,000; Dako Corporation; Carpinteria, CA) or rabbit anti-PRV antiserum (Rb133; 1:20,000; Dr. Lynn Enquist, Princeton University). Subsequent processing was performed at room temperature. Sections were rinsed in several changes of buffer, incubated for $1 \mathrm{hr}$ in biotinylated donkey anti-rabbit IgG (1:500; Jackson ImmunoResearch), rinsed, and incubated for $1.5 \mathrm{hr}$ in Vectastain Elite avidinbiotin reagents $(9 \mu \mathrm{l}$ "A" $+9 \mu \mathrm{l}$ "B" per $1.0 \mathrm{ml}$; Vector Laboratories, Burlingame, CA). After rinsing, sections were immersed for 3-5 min in a solution of $0.05 \%$ diaminobenzidine and $0.01 \% \mathrm{H}_{2} \mathrm{O}_{2}$ to generate a brown immunoperoxidase reaction product. Immunoreacted tissue sections were mounted onto Superfrost Plus glass slides (Fisher Scientific), dehydrated in graded ethanols, cleared in xylene, and coverslipped with Cytoseal 60 (Richard-Allan Scientific, Kalamazoo, MI). An adjacent set of sections was mounted onto slides and stained for Nissl substance with cresyl violet to clarify the anatomical location of H129 or PRV immunolabeling.

H129 was localized in celiac-superior mesenteric and nodose ganglia using immunofluorescence. For this purpose, a ring of rubber cement was laid down along the edges of each slide. Sections were covered with primary antibody solution made as described above, but at a 10-fold higher concentration. Slides were sealed in a humidified box and incubated overnight at room temperature. Slides were then rinsed and covered with a solution of secondary antiserum (Cy3-conjugated donkey anti-rabbit IgG; Jackson ImmunoResearch; 1:500) for $2 \mathrm{hr}$. After the rubber cement ring was removed, slides were rinsed, dehydrated, and coverslipped as described above.

Data analysis. Every sixth section (210 $\mu \mathrm{m}$ frequency) through the brainstem and forebrain, every fourth section (140 $\mu \mathrm{m}$ frequency) through the spinal cord, and every ganglia section was examined for immunolabeling by using a Zeiss Axiophot light microscope equipped for bright-field and fluorescent illumination. The distribution of immunolabeling was documented qualitatively in each experimental case, with reference as necessary to adjacent Nissl-stained sections to verify anatomical locations.

\section{Results}

\section{H129 transport from the stomach wall in intact rats}

Data from 21 surgically intact H129-injected rats were analyzed. Rats were killed after post-inoculation survival times of $72 \mathrm{hr}$ $(n=5), 96 \mathrm{hr}(n=6), 110 \mathrm{hr}(n=5)$, or $120 \mathrm{hr}(n=5)$. The central distribution of H129-immunolabeled profiles generally increased with increasing post-inoculation survival time, although the presence and extent of central H129 labeling varied among individual cases within each survival time group.

\section{Three-day survival}

Central infection was absent in the five rats killed $72 \mathrm{hr}$ after $\mathrm{H} 129$ injection. Infected neurons were present in the celiac-superior mesenteric and left nodose ganglia in three of these rats but were absent in the right nodose. In one rat, infected neurons were present in the celiac-superior mesenteric ganglion but were absent in the left and right nodose ganglia. No ganglionic infection was observed in the fifth rat. All five rats were asymptomatic at the time they were killed.

\section{Four-day survival}

Celiac-superior mesenteric and left nodose infection was observed in all six rats killed $96 \mathrm{hr}$ after H129 injection. Infected neurons were absent in the right nodose ganglia. Central infection (described below) was observed in four rats, two of which displayed adverse symptoms (i.e., nasal and lacrimal excretions, ungroomed coat, and $>10 \%$ loss of body weight). Central infection was absent in two rats, neither of which displayed adverse symptoms.

\section{Five-day survival}

Celiac-superior mesenteric, left nodose ganglia, and central infection were observed in all 10 rats killed either 110 or $120 \mathrm{hr}$ after H129 injection, and all rats displayed adverse symptoms of infection. A small number of infected neurons (i.e., 3-10 cells total per rat) were observed in the right nodose ganglia in three rats from the $120 \mathrm{hr}$ survival group.

\section{Central distribution of H129 labeling}

In intact rats with the most restricted central patterns of infection ( $n=4)$, H129-immunolabeled cells and fibers were present within the medullary dorsal vagal complex, thoracic spinal dorsal root entry zone, and thoracic spinal laminas I and II. Immunopositive fibers also were observed within the left tractus solitarius and medullary dorsal root entry zone of vagal afferent fibers and 

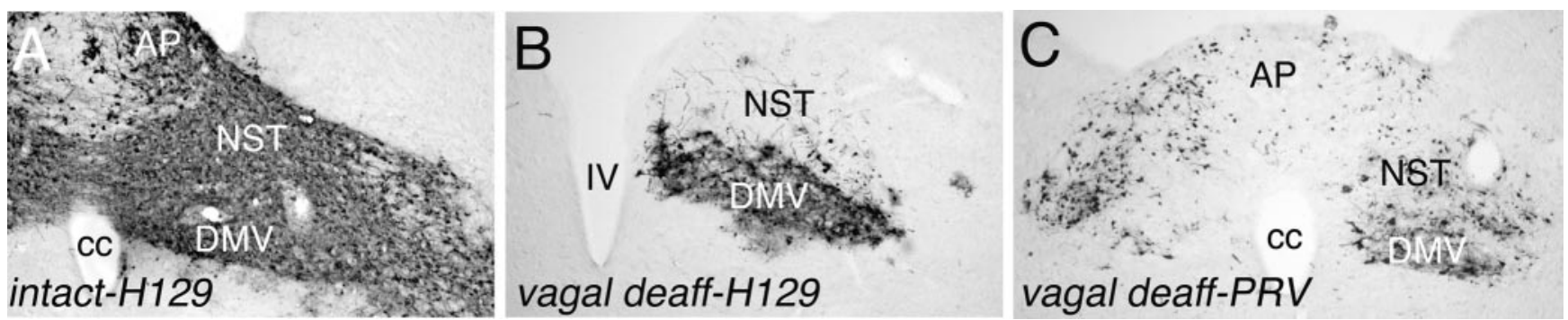

Figure 1. Immunoperoxidase labeling of transported $\mathrm{H} 129(A, B)$ or PRV $(C)$ in a surgically intact rat $(A)$ or in two rats after vagal deafferentation $(B, C)$. The deafferentation procedure eliminated anterograde transneuronal transport of $\mathrm{H} 129$ from the stomach wall into the NST and higher brain regions but did not interfere with retrograde transneuronal transport of PRV. AP, Area postrema; cc, central canal; DMV, dorsal motor vagal nucleus; NST, nucleus of the solitary tract; IV, fourth ventricle.

along the intramedullary path and ventral rootlet exit zone of vagal motor fibers. No other central regions contained viral immunolabeling at this early stage of infection. Spinal labeling was predominantly unilateral in each section examined, although the left and right sides of each section were not distinguished. In the medulla, early labeling occurred almost exclusively in the left dorsal vagal complex, where infected neurons were observed in both the NST and the dorsal motor nucleus of the vagus (DMV). Immunopositive cells with glial morphology often were present in regions that contained immunopositive neurons or fibers.

In intact rats with more widespread central patterns of infection, the overall density of H129-immunolabeled profiles increased within the dorsal vagal complex (Fig. $1 \mathrm{~A}$ ) and the thoracic dorsal horn and dorsomedial funiculus (Fig. $2 A$ ). Infected preganglionic sympathetic neurons never were observed in the thoracic intermediolateral cell column (Fig. 2A), although labeling was observed in additional spinal, brainstem, diencephalic, and telencephalic regions (Fig. 2). As transneuronal infection became more widely distributed, the density of H129 immunolabeling increased in previously infected regions. In rats with the most widespread infection, labeled brainstem areas included subregions of the spinal trigeminal nucleus and paratrigeminal area, medullary and pontine raphe nuclei, dorsal and ventral medullary reticular formation, spinocerebellar tract, cerebellum, medullary and pontine regions corresponding to the A1, A2, C1, C2, $\mathrm{C} 3, \mathrm{~A} 5, \mathrm{~A} 6$, and $\mathrm{A} 7$ catecholamine cell group regions, medial and lateral parabrachial nuclei (Fig. 2D), Barrington's nucleus and subcoeruleus region, pontine tegmental nuclei, and periaqueductal gray. Diencephalic labeling included neurons in the hypothalamic retrochiasmatic, tuberomammillary, perifornical, dorsomedial, paraventricular (Fig. 2B), supraoptic, arcuate, and lateral subnuclei (Fig. $2 E$ ), and in the dorsomedial thalamic paraventricular nucleus. Telencephalic labeling included neurons within the medial division of the central nucleus of the amygdala (Fig. 2C), zona incerta, substantia innominata, lateral and medial septal nuclei, dorsal and ventral subregions of the lateral bed nucleus of the stria terminalis (Fig. $2 F$ ), perirhinal cortex, and agranular insular cortex.

\section{Central H129 transport in vagally deafferented rats}

Data from seven vagally deafferented rats injected with H129 were analyzed. Rats were killed after post-inoculation survival times of $110 \mathrm{hr}(n=3)$ or $120 \mathrm{hr}(n=4)$. Only one of the rats killed at $110 \mathrm{hr}$ and two killed at $120 \mathrm{hr}$ displayed adverse symptoms, although central neural infection was observed in all seven rats. The distribution of spinal H129 labeling in vagal deafferented rats was similar to that observed in intact rats, described above. Conversely, brainstem labeling was markedly reduced in vagal deafferented rats, with labeled neurons confined almost
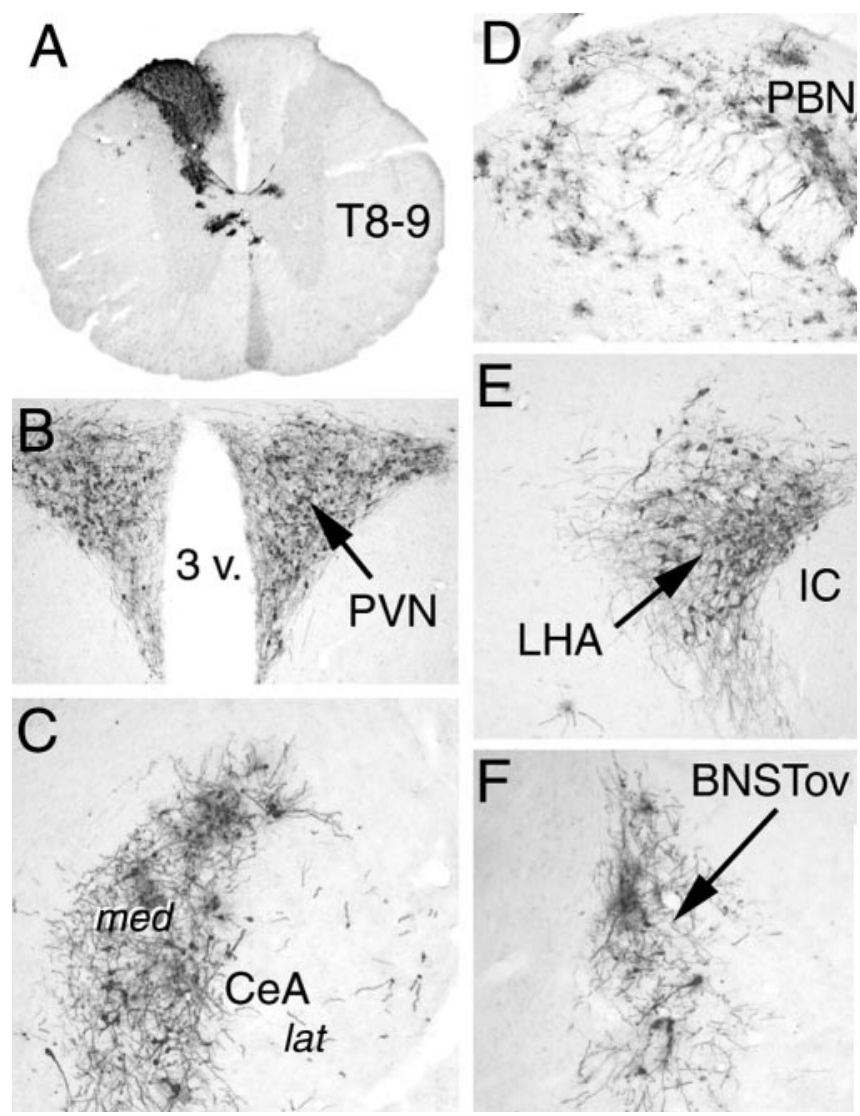

Figure 2. Immunoperoxidase labeling of H129 infection in a surgically intact rat after stomach wall inoculation. BNSTov, Bed nucleus of the stria terminalis, oval subnucleus; CeA, central nucleus of the amygdala, lateral (lat) and medial (med) divisions; IC, internal capsule; LHA, caudal lateral hypothalamic area; PBN, parabrachial nucleus; PVN, paraventricular nucleus of the hypothalamus; $78-9$, thoracic spinal cord, segment $8-9 ; 3 \mathrm{v}$., third ventricle.

exclusively to the left DMV (Fig. 1B). H129-labeled dendrites of infected DMV neurons were observed within the left medial NST (Fig. $1 B$ ). Pontine, diencephalic, and telencephalic labeling was absent in all vagal deafferented rats.

\section{Central PRV transport in intact and vagal deafferented rats}

The retrograde transneuronal viral tracer PRV (Bartha strain) was injected into the stomach wall in intact rats $(n=3)$ and vagal deafferented rats $(n=3)$ to determine whether the marked attenuation of central H129 labeling in deafferented rats (Fig. 1B) could be attributed to a general interference of the deafferentation procedure with central transneuronal viral transport. In vagally deafferented rats, labeled neurons within the DMV were 
confined almost exclusively to the left side (Fig. $1 C$ ). The overall distribution of retrograde transneuronal viral labeling in the spinal cord, NST, area postrema, and pre-autonomic forebrain nuclei (i.e., hypothalamus, amygdala, bed nucleus of the stria terminalis) was similar in intact and vagal deafferented rats and consistent with previous reports using the Bartha strain of PRV for stomach wall injections (Card et al., 1993; Yang et al., 1999; Rinaman et al., 2000).

\section{Discussion}

Central viscerosensory circuits receive continuous interoceptive feedback signals arising from within the body. These signals are conveyed to widespread regions of the CNS, including portions of the hypothalamus and limbic forebrain that are involved not only in modulating neuroendocrine and autonomic outflow but also in generating motivated behavior, controlling affect, and shaping emotional learning (Mayer and Saper, 2000). The present report is the first to examine the disynaptic and polysynaptic anatomical organization of central sensory pathways originating in a specific visceral organ (i.e., the stomach) and provides "proof of principle" for the utility and efficacy of anterograde transneuronal viral tracing in rats.

Sympathetic preganglionic neurons in the thoracic spinal cord were not infected after stomach wall injections of H129, providing strong initial evidence against retrograde transneuronal transport of this viral strain. Infected postganglionic sympathetic neurons were present, however, in the celiac-superior mesenteric ganglia, and infected preganglionic parasympathetic vagal motor neurons were present in the DMV. Vagal motor neuron infection can be accounted for, in part, by anterograde transneuronal transport of $\mathrm{H} 129$ via gastric vagal sensory terminals that synapse directly onto the dendrites of gastric vagal motor neurons (Rinaman et al., 1989); however, infection in the celiacsuperior mesenteric ganglion and some amount of vagal motor neuron labeling is most likely accounted for by first-order retrograde transport of $\mathrm{H} 129$ from the stomach wall injection sites. The axon terminals of vagal motor neurons synapse onto postganglionic neurons embedded in the stomach wall; thus, these axon terminals occupy the H129 injection sites, as do the axon terminals of celiac-superior mesenteric ganglion sympathetic neurons.

Experiments in vagal deafferented rats were performed to directly test the hypothesis that transneuronal transport of H129 within the CNS proceeds only in the anterograde direction. Most brain regions that receive ascending projections from the NST (Ter Horst et al., 1989; Ter Horst and Streefland, 1994) project reciprocally back to neurons located within the NST or DMV, or both (Loewy, 1990; Wallace et al., 1992). Thus, it was critical to demonstrate that transneuronal H129 infection in these higher brain regions could be eliminated by removal of vagal sensory inputs to the NST. Importantly, the vagal deafferentation procedure leaves vagal motor inputs to the ventral stomach wall intact and thus provides a rigorous test of transneuronal transport direction specificity. Indeed, first-order retrograde transport of H129 from the stomach wall injection site was evidenced by the presence of infected DMV neurons in vagal deafferented rats that lacked gastric vagal sensory inputs to the NST. Importantly, however, H129 was not transneuronally transported from infected vagal motor neurons to other central sites in vagal deafferented rats. In contrast, gastric DMV neurons and their sources of presynaptic input in the NST and other central sites were retrogradely and transneuronally infected as usual in vagal deafferented rats after PRV injections. We conclude that transneuronal transport of H129 from retrogradely infected autonomic motor neurons does not occur and thus does not contribute to the labeling observed in higher brainstem and forebrain regions in surgically intact rats.

The central patterns of transneuronal H129 infection observed in intact rats were generally consistent with the reported distribution of ascending projections from viscerosensory regions of the caudal NST (Ricardo and Koh, 1978; Sim and Joseph, 1994; Ter Horst and Streefland, 1994). The central terminals of gastric vagal afferents synapse initially within the medial and gelatinosus subnuclei of the NST (Shapiro and Miselis, 1985; Rinaman et al., 1989). Similarly, H129 labeling within the NST was initially relegated to fibers and cells in the solitary tract and the medial and gelatinosus subnuclei. Although gastric vagal sensory terminals are most dense within the gelatinosus subnucleus in rats, the region is so named because it contains relatively few neuron cell bodies. Instead, neurons with cell bodies in other NST subnuclei and the DMV send their dendrites into gelatinosus, where they receive synaptic inputs from gastric sensory afferents (Shapiro and Miselis, 1985; Rinaman et al., 1989). Other NST neurons and DMV neurons receive gastric vagal sensory input indirectly via local interneurons. Thus, it is not surprising that transneuronal anterograde transport of $\mathrm{H} 129$ proceeded to involve more widespread regions of the medial NST in rats killed at longer survival intervals. Previous tracing studies demonstrated that NST neurons project directly to the ventrolateral medulla, pontine parabrachial nucleus, hypothalamus, limbic forebrain, and other central regions (Saper and Loewy, 1980; Herbert et al., 1990). The present results confirm that these central viscerosensory projections include vagal sensory pathways that originate in the stomach wall.

Gastric spinal afferents terminate in laminas I, V-VII, and X of the mid-thoracic to upper lumbar spinal cord (Green and Dockray, 1987). Spinal viscerosensory fibers converge with somatic sensory fibers onto dorsal horn neurons that give rise to the ascending spinothalamic tract. A separate spinal pathway appears to relay viscerosensory signals through lamina $\mathrm{X}$, medial lamina VII, and the dorsal gray commissure, ascending at the junction of the gracile and cuneate fasciculi (Wang et al., 1999; Willis and Westlund, 2001). This pathway may merge with other ascending sensory pathways that access the forebrain by relaying in the caudal NST and medullary reticular formation (Menetrey and Basbaum, 1987; Potts et al., 2002), although direct spinal inputs to the parabrachial nuclei, hypothalamus, and amygdala also have been demonstrated (Menetrey and De Pommery, 1991). H129 tracing studies using longer survival times in bilaterally vagotomized rats will be necessary to reveal the central features of ascending spinal viscerosensory pathways and the extent to which they converge with ascending vagal sensory pathways.

The present results support the view that transneuronal transport of H129 through the rat CNS occurs exclusively in the anterograde direction; however, it is important to note that firstorder retrograde transport from peripheral inoculation sites also occurs. Neural circuit tracing with H129 can reliably identify medullary, pontine, cerebellar, mesencephalic, diencephalic, and telencephalic neurons that receive direct and relayed synaptic inputs originating from vagal and nonvagal afferents that serve the stomach and, theoretically, other visceral targets. We consider this tracing technique to be a novel and potentially powerful anatomical tool with which to identify multisynaptic central components of neural sensory pathways. 


\section{References}

Barnett EM, Evans GD, Sun N, Perlman S, Cassell MD (1995) Anterograde tracing of trigeminal afferent pathways from the murine tooth pulp to cortex using herpes simplex virus type I. J Neurosci 15:2972-2984.

Bartha A (1961) Experimental reduction of virulence of Aujeszky's disease virus. Magy Allotorv Lapja 16:42-45.

Card JP (1998) Exploring brain circuitry with neurotropic viruses: new horizons in neuroanatomy. Anat Rec 253:176-185.

Card JP, Rinaman L, Lynn RB, Lee B-H, Meade RP, Miselis RR, Enquist LW (1993) Pseudorabies virus infection of the rat central nervous system: ultrastructural characterization of viral replication, transport, and pathogenesis. J Neurosci 13:2515-2539.

Dix RD, McKendall RR, Baringer JR (1983) Comparative neurovirulence of herpes simplex type 1 strains after peripheral or intracerebral inoculation of BALB/c mice. Infect Immun 40:103-112.

Green T, Dockray GJ (1987) Calcitonin gene-related peptide and substance $\mathrm{P}$ in afferents to the upper gastrointestinal tract in the rat. Neurosci Lett 76:151-156.

Herbert H, Moga MM, Saper CB (1990) Connections of the parabrachial nucleus with the nucleus of the solitary tract and the medullary reticular formation in the rat. J Comp Neurol 293:540-580.

Kelly RM, Strick PL (2003) Cerebellar loops with motor cortex and prefrontal cortex of a nonhuman primate. J Neurosci 23:8432-8444.

Loewy AD (1990) Central autonomic pathways. In: Central regulation of autonomic functions (Loewy AD, Spyer KM, eds), pp 88-103. New York: Oxford UP.

Loewy AD (1995) Pseudorabies virus: a transneuronal tracer for neuroanatomical studies. In: Viral vectors. Gene therapy and neuroscience applications (Loewy AD, Kaplitt MG, eds), pp 349-366. San Diego: Academic.

Mayer EA, Saper CB, eds (2000) The biological basis for mind body interactions. Amsterdam: Elsevier.

McLean IW, Nakane PK (1974) Periodate-lysine-paraformaldehyde fixative. A new fixative for immunoelectron microscopy. J Histochem Cytochem 22:1077-1083.

Menetrey D, Basbaum AI (1987) Spinal and trigeminal projections to the nucleus of the solitary tract: a possible substrate for somatovisceral and viscerovisceral reflex activation. J Comp Neurol 255:439-450.

Menetrey D, De Pommery J (1991) Origins of spinal ascending pathways that reach central areas involved in visceroception and visceronociception in the rat. Eur J Neurosci 3:249-259.

Norgren R, Smith GP (1994) A method for selective section of vagal afferent or efferent axons in the rat. Am J Physiol Regul Int Comp Physiol 267:R1136-R1141.

Potts JT, Lee SM, Anguelov PI (2002) Tracing of projection neurons from the cervical dorsal horn to the medulla with the anterograde tracer biotinylated dextran amine. Auton Neurosci Basic Clin 98:64-69.

Ricardo JA, Koh ET (1978) Anatomical evidence of direct projections from the nucleus of the solitary tract to the hypothalamus, amygdala, and other forebrain structures in the rat. Brain Res 153:1-26.

Rinaman L, Card JP, Schwaber JS, Miselis RR (1989) Ultrastructural demonstration of a gastric monosynaptic vagal circuit in the nucleus of the solitary tract in rat. J Neurosci 9:1985-1996.

Rinaman L, Levitt P, Card JP (2000) Progressive postnatal assembly of limbic-autonomic circuits revealed by central transneuronal transport of pseudorabies virus. J Neurosci 20:2731-2741.

Saper CB, Loewy AD (1980) Efferent connections of the parabrachial nucleus in the rat. Brain Res 197:291-317.

Schwartz GJ, Salorio CF, Skoglund C, Moran TH (1999) Gut vagal afferent lesions increase meal size but do not block gastric preload-induced feeding suppression. Am J Physiol Regul Int Comp Physiol 276:R1623-R1629.

Shapiro RE, Miselis RR (1985) The central organization of the vagus nerve innervating the stomach of the rat. J Comp Neurol 238:473-488.

Sim LJ, Joseph SA (1994) Efferents of the opiocortin-containing region of the commissural nucleus tractus solitarius. Peptides 15:169-174.

Strack AM, Sawyer WB, Hughes JH, Platt KB, Loewy AD (1989) A general pattern of CNS innervation of the sympathetic outflow demonstrated by transneuronal pseudorabies viral infections. Brain Res 491:156-162.

Sun N, Cassell MD, Perlman S (1996) Anterograde, transneuronal transport of herpes simplex virus type 1 strain H129 in the murine visual system. J Virol 70:5405-5413.

Ter Horst GJ, Streefland C (1994) Ascending projections of the solitary tract nucleus. In: Nucleus of the solitary tract (Barraco IRA, ed), pp 93-103. Boca Raton, FL: CRC.

Ter Horst GJ, Boer PD, Luiten PGM, Willigen JDV (1989) Ascending projections from the solitary tract nucleus to the hypothalamus. A Phaseolus vulgaris lectin tracing study in the rat. Neuroscience 31:785-797.

Wallace DM, Magnuson DJ, Gray TS (1992) Organization of amygdaloid projections to brainstem dopaminergic, noradrenergic, and adrenergic cell groups in the rat. Brain Res Bull 28:447-454.

Wang C-C, Willis WD, Westlund KN (1999) Ascending projections from the area around the spinal cord central canal: a Phaseolus vulgaris leucoagglutinin study in rats. J Comp Neurol 415:341-367.

Watson RE, Wiegand ST, Clough RW, Hoffman GE (1986) Use of cryoprotectant to maintain long-term peptide immunoreactivity and tissue morphology. Peptides 7:155-159.

Willis Jr WD, Westlund KN (2001) The role of the dorsal column pathway in visceral nociception. Curr Pain Headache Rep 5:20-26.

Yang M, Card JP, Tirabassi RS, Miselis RR, Enquist LW (1999) Retrograde, transneuronal spread of pseudorabies virus in defined neuronal circuitry of the rat brain is facilitated by gE mutations that reduce virulence. J Virol 73:4350-4359.

Zemanick MC, Strick PL, Dix RD (1991) Direction of transneuronal transport of herpes simplex virus 1 in the primate motor system is straindependent. Proc Natl Acad Sci USA 88:8048-8051. 